(c) American Dairy Science Association, 2007.

\title{
Nonparametric Analysis of the Impact of Inbreeding on Production in Jersey Cows
}

\author{
D. Gulisija, ${ }^{1}$ D. Gianola, and K. A. Weigel \\ Department of Dairy Science, University of Wisconsin, Madison 53706
}

\begin{abstract}
Under dominance, the relationship between a quantitative trait and the inbreeding coefficient $(F)$ is expected to be linear. Epistasis involving dominance effects or selection against inbred individuals can produce nonlinear patterns in the form of inbreeding depression. The form of the relationship between $F$ and yield traits was explored via local regression (LOESS). First-lactation milk, fat, and protein records from 59,778 Jersey cows with at least 6 generations of known pedigree were used. The $F$ ranged from 0.6 to $34 \%$ and median $F$ was $6.3 \%$. The LOESS regressions of predicted residuals from an animal model (empirical best linear unbiased predictions, EBLUP) on $F$ were calculated for each trait; the EBLUP model included fixed herd-year-season, age at calving and DIM effects, and random additive genetic effects. The relationship between EBLUP residuals and inbreeding was complex and nonlinear. Yields were unaffected for $F \leq 7 \%$, and inbreeding depression seemed to stabilize at $F>20 \%$ for fat and protein yield. For SCS, both parametric and LOESS fits were consistent with the absence of sizable dominance effects. Effects of inbreeding on performance seemed to be more complex than suggested by previous studies based on linear regression. Results should be interpreted with caution because the data were scarce at high levels of $F$.
\end{abstract}

Key words: nonparametric regression, inbreeding, Jersey cattle

\section{INTRODUCTION}

As the inbreeding coefficient ( $F$; Wright, 1922; Malécot, 1948) increases, mean values of quantitative traits typically decline, and this is termed inbreeding depression (Falconer and Mackay, 1996; Lynch and Walsh, 1998). Under dominance, which is thought to account for most of the inbreeding depression (Charlesworth and Charlesworth, 1987), the mean of a quantitative trait can be expressed as a linear function of $F$.

Received December 20, 2005.

Accepted June 12, 2006.

${ }^{1}$ Corresponding author: gulisija@calshp.cals.wisc.edu
Epistasis can also contribute to inbreeding depression (Crow and Kimura, 1970; Bulmer, 1980; Hill, 1982; Lynch, 1991), even though it is thought to play a small or negligible role (Falconer and Mackay, 1996). If dominance interactions involve 2 or more loci, the mean value can be expressed as a high-order polynomial in $F$ (Crow and Kimura, 1970; Lynch and Walsh, 1998). However, only small departures from linearity are expected, because the high-order terms in $F$ tend to be small, especially when $F$ is low (Mackay, 2001). If epistatic effects are sizable, the rate of decline in mean value with respect to $F$ can either diminish as inbreeding increases (i.e., diminishing epistasis) or accelerate with respect to $F$ (i.e., reinforcing epistasis).

Inbreeding depression due to unfavorable recessive alleles with major effects can be effectively reduced or purged via selection against inbred individuals, or by random drift (Hedrick, 1994; Glemin, 2003; Gulisija and Crow, accepted). Thus, some of the highly inbred individuals could be removed from a population before reaching productive age. This selection process can produce nonlinearity in the relationship between a trait and the inbreeding level. Purging of deleterious alleles would be reflected by a reduced rate of decline in performance at higher levels of inbreeding (resembling diminishing epistasis).

Numerous studies have used linear-regression to estimate inbreeding depression for production in dairy cattle (e.g., Allaire and Henderson, 1965; Hudson and Van Vleck, 1984; VanRaden et al., 1992; Miglior et al., 1995b; Smith et al., 1998), but evidence of effects of inbreeding on SCC is scarce (Miglior et al., 1995a; Smith et al., 1998). Several studies in the animal breeding field have addressed the form of the relationship between quantitative traits and $F$. Departures from linearity at higher levels of $F$ were indicated in dairy cattle (Hudson and Van Vleck, 1984; Miglior et al., 1992; Thompson et al., 2000b; Wall et al., 2005) and in horses (Klemetsdal, 1998). These studies suggested a faster depression rate at higher values of $F$, with little recovery at the highest levels of inbreeding. Although Thompson et al. (2000a) obtained similar results for 305-d milk yield, no systematic departure from linear inbreeding depression was found for fat and protein 
yield in US Holsteins. Previous studies suggest that the relationship between quantitative traits and the inbreeding coefficient might be more complex than would be expected from a genetic model with additive and dominance effects only.

The objective of this research was to examine the impact of inbreeding on SCS and on milk, protein, and fat yields in US Jersey cows using nonparametric regression models.

\section{MATERIALS AND METHODS}

\section{Data}

Pedigree records of 185,223 US Jersey cows, with first calving in 1995 through 2000, and of their ancestors, were extracted from the American Jersey Cattle Association database. Pedigrees with obvious errors (e.g., animals with dates of birth prior to those of their ancestors) were removed. The edited pedigree file included 500,729 animals born from 1923 through 1998. Inbreeding coefficients were computed using the PROC INBREED procedure (SAS Institute, 2002). In view of the fact that the inbreeding coefficient value depends on the depth of the pedigree, and to reduce errors caused by missing pedigree information, a subset of data (59,778 records) containing only cows with at least 6 generations of known pedigree was formed and used in the statistical analysis.

\section{Statistical Models}

A 2-stage analysis was performed. First, empirical best linear unbiased predictions (EBLUP) of residuals in models that accounted for additive genetic effects, with or without $F$ as a covariate, were obtained. In the second stage, EBLUP were studied via a nonparametric regression method (LOESS), described later.

Inbreeding depression is expected to be a function of dominance effects and of interactions involving dominance effects. The assumption in this study was that, working with the EBLUP residuals, the analysis would become net of additive effects, which do not contribute to inbreeding depression. Although the entire analysis can be performed in a single stage, the 2-step approach conferred computational flexibility, because varying forms of inbreeding depression could be explored in a local regression context (in the second stage). The EBLUP residuals from models accounting for $F$ were studied to assess the goodness of fit.

In the first stage, 4 univariate linear models were fitted to first-lactation SCS and milk, fat, or protein yield (305-d mature equivalent) records. Model 1 was

$$
\mathbf{y}=\mathbf{X} \boldsymbol{\beta}+\mathbf{Z u}+\mathbf{e},
$$

where $\mathbf{y}$ is an $\mathrm{n} \times 1$ vector of SCS and milk, protein, or fat yields; $\mathbf{X}$ and $\mathbf{Z}$ are incidence matrices of order $\mathrm{n} \times$ $\mathrm{p}$ and $\mathrm{n} \times \mathrm{q}$, respectively; $\boldsymbol{\beta}$ is a $\mathrm{p} \times 1$ vector of fixed effects; and $\mathbf{u}$ is a $q \times 1$ vector of random additive genetic effects for all the animals in the pedigree, with $\mathrm{q}=$ 266,470 . The $\beta$ vector included herd-year-season effects $(6,406$ levels, with season classes of January to April, May to August, and September to December), age at calving effects (6 levels: $<617,617$ to 716,717 to 816 , 817 to 916,917 to 1,016 , or $>1,016 \mathrm{~d}$ of age), and a regression coefficient on DIM deviation from the average $(\bar{D}=263)$, and $\mathbf{e}$ is an $\mathrm{n} \times 1$ vector of random residuals, with the typical element $e_{i j k}$ (corresponding to cow $k$ calving in herd-year-season $i$ at age $j$ ). Although yields were 305-d mature equivalent, DIM had a small but significant effect. Because there was no colinearity between $F$ and DIM, this covariate was included in the model.

Genetic and residual effects were assumed to be mutually independent, with $\mathbf{u} \sim \mathrm{N}\left(\mathbf{0}, \mathbf{A} \sigma_{a}^{2}\right)$ and $\mathbf{e} \sim$ $\mathrm{N}\left(\mathbf{0}, \mathbf{I} \sigma_{e}^{2}\right)$, where $\mathbf{A}$ is the additive relationship matrix (containing $1+F_{i}$ in the $i$ th diagonal position, where $F_{i}$ is the inbreeding coefficient of animal $i$ ), $\mathbf{I}$ is an identity matrix of order equal to the number of records, and $\sigma_{a}^{2}$ and $\sigma_{e}^{2}$ are additive genetic and residual components of variance, respectively.

Model 2 was like model 1, but it also had a linear regression of performance on $F$, such that $\beta$ included a linear regression coefficient of performance on inbreeding level $\left(F_{k}=\right.$ inbreeding coefficient of cow $\left.k\right)$. Model 3 was like model 2, plus a quadratic regression coefficient of performance on $F$. Last, model 4 included a cubic effect, in addition to the first- and secondorder terms.

Variance components were estimated by univariate REML with the program REMLF90 (http://nce.ads. uga.edu/ ignacy/programs.html) for each of the 4 traits. Using the REML estimates as true parameter values, mixed-model equations were solved using the program BLUPF90 (http://nce.ads.uga.edu/ ignacy/programs. html), to obtain estimates of model effects. The EBLUP of residuals were then computed as

$$
\hat{\mathbf{e}}=\mathbf{y}-\mathbf{X} \hat{\boldsymbol{\beta}}-\mathbf{Z} \hat{\mathbf{u}} .
$$

\section{Local Regression Analysis with LOESS}

The EBLUP residuals $\left(\hat{e}_{i j k}\right)$, which were expected to be net of effects of location parameters and of additive genetic values, were modeled via local regression using $F$ as an explanatory variable. The objective was to assess the extent to which models 1 to 4 adequately de- 
scribed the relationship between performance and inbreeding.

Local regression is a nonparametric approach to fitting curves and surfaces to data based on smoothing (Cleveland and Loader, 1996). The local regression method used, LOESS (Cleveland, 1979; Cleveland and Devlin, 1988; Cleveland et al., 1992), approximates the relationship between response and explanatory variables locally by a smooth curve based on a parametric function, using locally weighted least squares. Weights are assigned such that points close (Euclidean distance) to the predictor value $(F)$ of interest receive higher weight. Also, a subset of the data can be used in the local fit. Subsets are distinct across neighborhoods, constructed such that values of the predictor variables are close to the focal predictor value of interest. Neighborhood size is defined via a spanning parameter $(f) ; f=1$ implies that all data points are used in the local fit, and a spanning parameter $<1$ denotes that only a subset $(f \times 100 \%)$ of the points is used in the local fit. A smaller spanning parameter allows more detailed assessment of local behavior but increases variance. Once local predictions are obtained for each given predictor, predicted values are returned, and a smoothed curve is formed. In our context, LOESS allows discovery of ranges of $F$ values where inbreeding depression differs from what would be expected based on global fit.

As opposed to splines, which are frequently used for smoothing, LOESS is arguably more robust, because it is less influenced by "knot-to-knot" polynomial fit. As with splines, the LOESS method can also be embedded into mixed-model methodology (i.e., semiparametric models). However, LOESS and EBLUP residuals were used in this study, because a 2 -step approach provided more opportunities to explore local behaviors in a flexible and computationally feasible manner.

$F$-Statistics were computed to test whether LOESS improved fit over first-order regression on $F$ for EBLUP residuals from model 1 . This is valid, because parametric and nonparametric models are properly nested; a linear relationship is a special case of a general, potentially nonlinear, relationship (Fox, 2005).

A LOESS analysis can be affected by outliers or by data structure, such as scarcity of observations at high values of $F$. In addition to regular LOESS curves, robust LOESS fits for production traits were obtained using the entire data set. In robust LOESS, which is designed to attenuate outliers, weights are assigned through an iterative process that penalizes data points that are far away from their predicted values. To avoid misleading inference due to data structure, and to explore different values of spanning parameters, medians of EBLUP residuals were computed at each value of $F$ (1,622 distinct $F$ values at 4 decimal digits). These medians were used for robust LOESS fit at optimal values of the spanning parameter $f$. Optimal values of $f$ were assessed via cross-validation. The cross-validation function, $C V(f)$, was the average of squared differences of values at focal data points and predictions from a fit obtained without using the focal data point (Fox, 2005). $C V(f)$ was evaluated at $f$ values ranging from 0.1 to 1 (by 0.1 increments), with the optimal $f$ being the minimizer of this function.

To assess uncertainty associated with the LOESS fit, a simple bootstrap approach was used (Efron, 2005). Here, the 1,622 medians of the EBLUP residuals were resampled with replacement and 100 bootstrap samples were obtained. For each bootstrap sample, a robust LOESS curve was fitted, thus creating a family of 100 regression curves. Computations utilized the $\mathrm{R}$ software (R Development Core Team, 2004).

\section{RESULTS AND DISCUSSION}

\section{Degree of Inbreeding}

In the sample of cows selected for the analysis, $F$ ranged from 0.6 to $34 \%$, and the distribution of $F$ values had a longer tail to the right (Figure 1). Mean and median $F$ were 6.8 and $6.3 \%$, respectively, with the 25 th and 75 th percentiles of the distribution at 4.5 and $8.3 \%$, respectively. All cows had some inbreeding, and 33\% of the animals had $F<5 \%$. Most animals had $F<10 \%$ (86.9\%), and only 1,266 cows $(2.1 \%)$ had $F$ larger than $15 \%$.

\section{Parametric Regression}

Heritability estimates reported are from model 1, but were consistent across models. Estimates were 0.34,

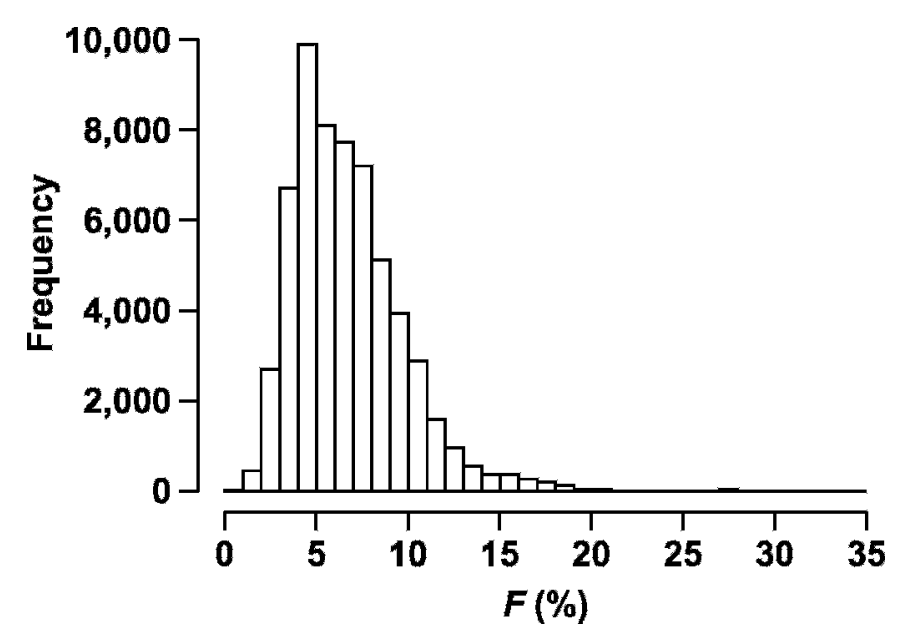

Figure 1. Frequency distribution of inbreeding coefficients $(F)$ for cows with at least 6 generations of known pedigree data. 
Table 1. Estimates of regression coefficients (units of additive genetic standard deviation, $\sigma_{\mathrm{a}}$ ) for linear, quadratic, and cubic terms in $F(\%)$

\begin{tabular}{|c|c|c|c|c|c|}
\hline Trait $^{1}$ & Model & $\begin{array}{l}\text { Covariate } \\
F(\%)\end{array}$ & $F^{2}(\%)$ & $F^{3}(\%)$ & $\sigma_{\mathrm{a}}$ \\
\hline \multicolumn{6}{|l|}{ Milk } \\
\hline & Linear & -0.0459 & & & \multirow{3}{*}{$\begin{array}{l}637.5 \mathrm{~kg} \\
\left(\mathrm{~h}^{2}=0.34\right)\end{array}$} \\
\hline & Quadratic & -0.0656 & 0.000989 & & \\
\hline & Cubic & -0.0334 & -0.002120 & 0.00007825 & \\
\hline \multirow{3}{*}{ Fat } & Linear & -0.0561 & & & \multirow{3}{*}{$\begin{array}{c}21.6 \mathrm{~kg} \\
\left(\mathrm{~h}^{2}=0.22\right)\end{array}$} \\
\hline & Quadratic & -0.0834 & 0.001470 & & \\
\hline & Cubic & -0.0496 & -0.001890 & 0.00008402 & \\
\hline \multirow{3}{*}{ Protein } & Linear & -0.0540 & & & \multirow{3}{*}{$\begin{array}{c}17.7 \mathrm{~kg} \\
\left(\mathrm{~h}^{2}=0.26\right)\end{array}$} \\
\hline & Quadratic & -0.0771 & 0.001156 & & \\
\hline & Cubic & -0.0532 & -0.001169 & 0.00006210 & \\
\hline \multirow{3}{*}{ SCS } & Linear & 0.0059 & & & \multirow{3}{*}{$\begin{array}{c}0.44 \\
\left(h^{2}=0.15\right.\end{array}$} \\
\hline & Quadratic & 0.0235 & -0.000882 & & \\
\hline & Cubic & 0.0114 & 0.000293 & -0.00003147 & \\
\hline
\end{tabular}

${ }^{1}$ Trait $=305-d$ mature equivalent.

0.22 , and 0.26 for milk, fat, and protein yields, respectively. Estimated heritability of SCS was 0.15. Estimates of regression coefficients for linear, quadratic, and cubic effects of $F$ are given in Table 1, in units of $\sigma_{a}$ (estimated from model 1). Somatic cell score did not exhibit detectable inbreeding depression, irrespective of the order of the polynomial fitted $(P>0.05)$. Similar results were reported by Smith et al. (1998) and Thompson et al. (2000b). Miglior et al. (1995a) did find an effect of 0.012 units per percentage of $F$, which was small. These findings suggest that for SCS, the effect of dominance is rather small or absent.

Estimated linear regressions on $F$ were negative for yield traits throughout, indicating inbreeding depression. Coefficients pertaining to higher order terms in $F$ were generally small, although these coefficients can have a large impact at high values of $F$. The quadratic and cubic coefficients depict changes in the inbreeding depression as $F$ varies. For instance, a plot of fitted values obtained from the quadratic and cubic models (not shown) suggested that inbreeding acted nearly linearly at low values of $F$ but curvilinearly at high values of $F$, for yield traits.

In the quadratic model, second-order regressions on $F$ were positive for yield traits, suggesting a diminishing rate of inbreeding depression. Furthermore, the inbreeding depression seemed to stabilize at high values of $F$. This could reflect diminishing epistasis or partial purging of deleterious alleles by selection.

In the third-order model (Table 1), coefficients corresponding to linear and quadratic terms in $F$ were negative for yield traits, whereas cubic terms were positive. Partial recovery (perhaps due to purging of deleterious genes) was suggested for milk yield at $F>25 \%$. A simi- lar pattern was observed for fat and protein yields, where a diminishing rate of depression occurred at high values of $F$.

To examine whether models 2 to 4 fitted the data better than model 1, the EBLUP of residuals from model 1 and 3 models including $F$ as a covariate in the explanatory structure were analyzed via simple linear and nonparametric regression. Regressions of EBLUP residuals from model 1 with inbreeding coefficient as a covariate indicated that third-order regression on $F$ improved fit significantly over models with lesser order regression on $F$ for yield traits $(P<0.01$, Table 2$)$. Robust LOESS curves of EBLUP residuals from all models supported these findings and are discussed subsequently.

\section{LOESS Analyses of EBLUP Residuals from Model 1}

LOESS improved the fit over that of the first-order regression on $F$ for yield traits $(P<0.05)$, but not for SCS $(P>0.05)$, which was unaffected by inbreeding. Because SCS did not exhibit inbreeding depression with either parametric or nonparametric fits, this trait was omitted from further analyses.

The LOESS curves for yield traits from the model ignoring $F$ (model 1) are shown in Figure 2. These curves, using the entire data $(f=1)$ with a local weighted least squares fit, were obtained using a second-degree local polynomial for both the regular and robust fits. In general, LOESS suggested nonlinear relationships between yield traits and $F$.

With the regular LOESS fit, milk yield did not decrease until $F=7 \%$, and decreased subsequently. Fitted curves were strictly decreasing for milk and protein yields. Protein yield exhibited a smaller rate of decrease 
Table 2. $F$-statistics ( $P$-values) used to determine best fit among models with null, first-, second-, or thirdorder regressions of EBLUP residuals (model 1) on $F$

\begin{tabular}{llcc}
\hline & $F_{1,59777}(P$-value $)$ & $F_{1,59776}(P$-value $)$ & $F_{1,59775}(P$-value $)$ \\
Trait $^{1}$ & $F$ vs. no effect & $F+F^{2}$ vs. $F$ & $F+F^{2}+F^{3}$ vs. $F+F^{2}$ \\
\hline Milk & $22.81(<0.001)$ & $89.99(<0.001)$ & $31.59(<0.001)$ \\
Fat & $30.54(<0.001)$ & $96.09(<0.001)$ & $50.64(<0.001)$ \\
Protein & $36.10(<0.001)$ & $114.95(<0.001)$ & $53.64(<0.001)$ \\
SCS & $0.10(0.75)$ & $0.007(0.93)$ & $1.51(0.22)$ \\
\hline
\end{tabular}

${ }^{1}$ Trait $=305-\mathrm{d}$ mature equivalent.

after $F \approx 20 \%$, whereas minor departures from linearity were noted for milk yield. For fat yield, inbreeding depression also seemed to stabilize at high levels of inbreeding, because no further depression was observed for $F>23 \%$.

Once outliers were attenuated via the robust fits, milk yield did not decrease until $F \geq 9 \%$. Inbreeding depression appeared to be less severe throughout the range of $F$ with the robust LOESS fit. In general, regular and robust LOESS had similar forms, but with some discrepancies at the higher levels of $F$. For example, with robust LOESS, protein stabilized at higher levels of $F$ than that observed with regular LOESS. Also, fat yield recovered at a smaller $F(24 \%)$ with robust LOESS, which did not happen with the regular fit. Milk yield followed a similar trajectory as that observed in the regular fit.

The small discrepancies between the regular and robust LOESS analysis could be an artifact of data structure. However, it is possible that a few rare alleles

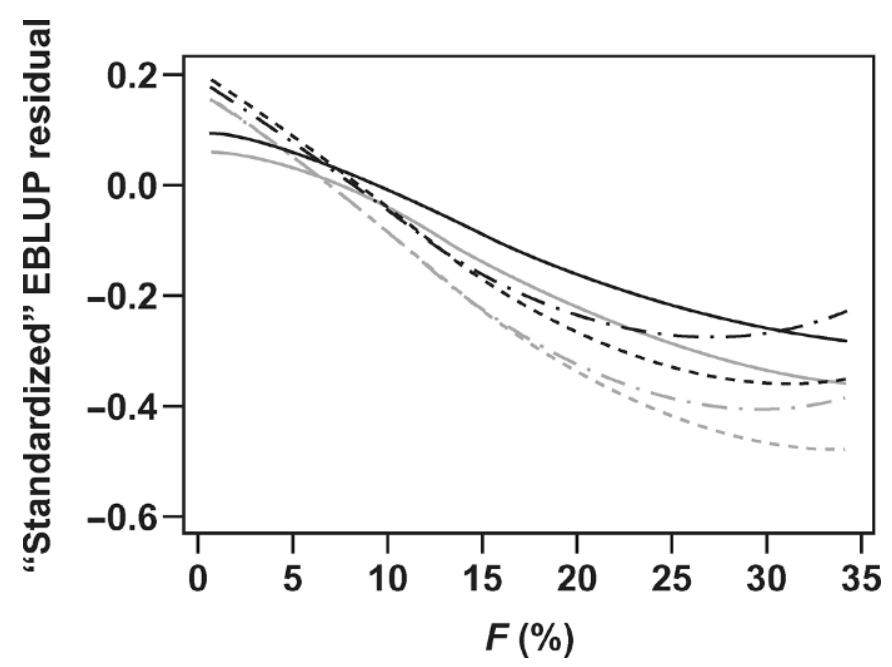

Figure 2. Regular (gray) and robust (black) LOESS curves for lactation milk ( $(-)$, fat (- - - -), and protein (- - -) yields based on empirical best linear unbiased prediction (EBLUP) residuals $(y$ axis $=\hat{e}_{i j k} / \hat{\sigma}_{a}$ ) from the first stage of the analysis (second-degree local polynomial and spanning parameter $f=1$ used in LOESS fitting). $F=$ inbreeding coefficient. with strong effects may underlie the differences. The observed curvature in the 2 analyses may indicate that animals having higher levels of $F$ are perhaps survivors of a purging process. Selection could have removed genetic load prior to production. Thus, the interplay of selection and inbreeding can distort the picture of the inbreeding depression.

Hudson and Van Vleck (1984), Miglior et al. (1992), Thompson et al. (2000b), and Wall et al. (2005) also reported nonlinearity in inbreeding depression. Wall et al. (2005) observed a lesser effect until $F=8 \%$, in agreement with our study. However, other studies cited seem to suggest a faster depression rate at higher values of $F$, with little recovery at the highest levels of inbreeding. Hudson and Van Vleck (1984) and Miglior et al. (1992) associated recovery with involuntary culling and sparse data structure. However, except for the study of Wall et al. (2005), in the cited studies pedigree data was not edited as stringently as in this study. It is possible that with stringent data editing, cows were selected with more generations of inbred ancestors since the base. Thus, it is possible that the analyzed sample consisted of animals that had, on average, more opportunity for purging than an average cow.

Miglior et al. (1994) found between-family heterogeneity in inbreeding depression for production traits in a group of Holstein cattle. Also, Gulisija et al. (2006) found between-founder heterogeneity in inbreeding depression for production traits from the sample of cows used in this study. These findings are important because the variability among cattle families implies that relatively few alleles with major effects are involved in inbreeding depression. Major-effect alleles can be effectively selected against in homozygotes, and this is the basic mechanism of purging. Gulisija and Crow (accepted) showed that the inbreeding decline due to major factors is reduced by about $12.6 \%$ because of ancestral inbreeding in the group of cows used in this study. Given that the opportunity of purging increased with $F$ for the sample of animals used in analyses (correlation $=0.93$, unpublished results), diminishing effects of $F$ at the higher levels were expected. 

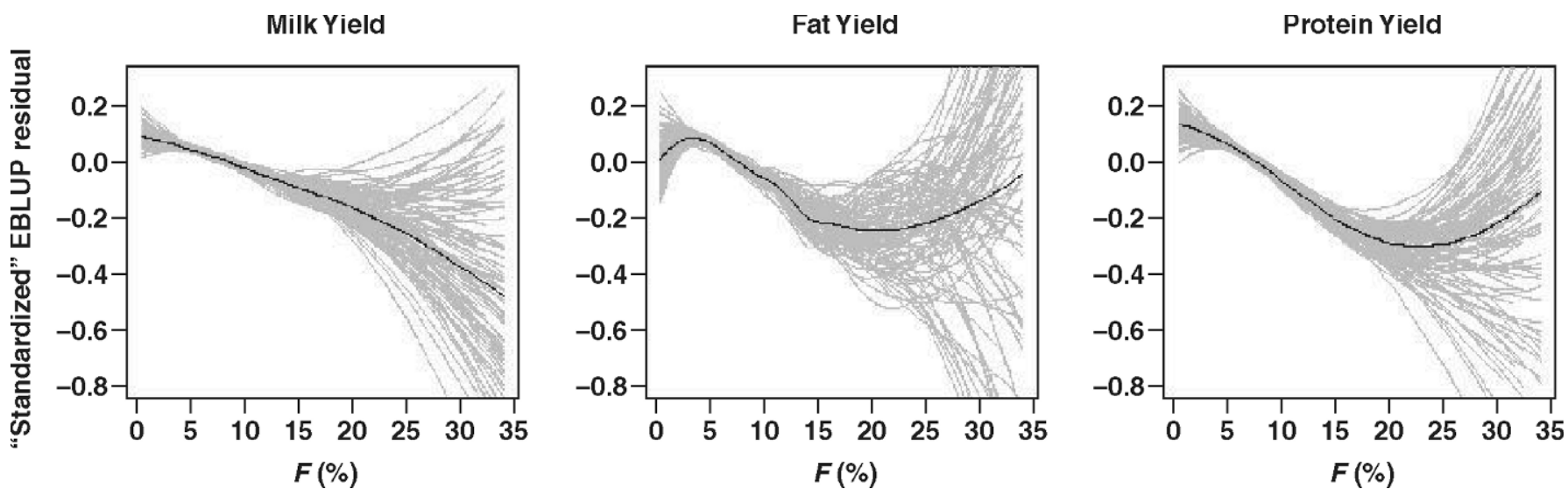

Figure 3. Robust original (black) with bootstrap (gray) LOESS curves for lactation milk $(f=0.9)$, fat $(f=0.5)$, and protein $(f=0.9)$ yields based on medians of empirical best linear unbiased predictions (EBLUP) residuals $\left(y\right.$-axis $\left.=\hat{e}_{i j k} / \hat{\sigma}_{a}\right)$ from the first stage of the analysis (second-degree local polynomial used in LOESS fitting). $F$ = inbreeding coefficient.

\section{Robust LOESS Analyses of Medians of EBLUP Residuals from Model 1}

Results from the LOESS analysis may have been affected by data structure. For instance, while LOESS curves for yields were decreasing (Figure 2), only 35 cows had $F<1 \%$, and the data set was sparse for $F>$ 15\% (Figure 1). Hence, LOESS predictions for highly inbred animals might be strongly influenced by residuals from animals with small $F$. To reduce possible distortions caused by an uneven data structure, medians of EBLUP residuals were computed for each unique $F$ value, in the sense mentioned earlier.

Figure 3 gives LOESS curves obtained with $f=0.9$, 0.5 , and 0.9 (optimal values) and a second-degree local polynomial, along with bootstrapped LOESS curves for milk, fat, and protein yields, respectively. The robust LOESS curve for milk yield was strictly decreasing, and at a faster rate at higher inbreeding levels. The fat yield curve increased slightly at small levels of $F(F<$ $4 \%$ ), as opposed to the strictly decreasing curves found with the entire data. After a drop for $F$ values between 8 and $15 \%$, fat and protein yields did not show further depression at higher levels of $F$, but rather yields increased. However, statistical variability was very large at very small values of inbreeding, and especially at $F>15 \%$, making it impossible to draw any definitive conclusions outside of a range of $F$ between 5 and $15 \%$.

\section{Robust LOESS Analyses of EBLUP Residuals from Models with Regressions on F}

The EBLUP of residuals for the 3 models including $F$ as a covariate in the explanatory structure were also analyzed, to examine whether models 2 to 4 fitted the data better than model 1 . Robust LOESS curves of
EBLUP residuals from these models obtained using the entire data $(f=1)$ for a local weighted least squares fit with a second-degree local polynomial are given in Figure 4.

For milk, fat, and protein yields, LOESS curves of residuals from models 2 and 3 were essentially flat for smaller to moderate values of $F$ and increased thereafter. Hence, lack of fit was clear for data from highly inbred animals. Model 2 (linear in $F$ ) had the worst fit among the 3 models. As shown in Figure 4, standardized residuals from this model increased for yield traits for values of $F$ larger than $10 \%$. In the model with $F^{2}$, lack of fit was still apparent for values of $F$ larger than $20 \%$. Inbreeding depression for yield traits seemed to be overestimated by models 2 and 3 for animals with higher $F$, because predicted values were smaller than actual yields. Model 4 (with terms in $F, F^{2}$, and $F^{3}$ ) had the best fit among the models considered, with only mild departures observed from a horizontal line. This also implies that performance varies nonlinearly with $F$. Assuming that the cubic model (model 4) holds, and using the estimates in Table 1 for milk yield, the rate of change of milk yield with respect to inbreeding would be $d \mathrm{milk} / d \mathrm{~F}=-0.034-0.00414 F+0.00023475 F^{2}$. Setting this expression to 0 and solving it gives 2 real roots: $F_{1}=-6.10$ and $F_{2}=23.74$. It can be verified that this derivative is negative (but rather flat) from $F=0$ until $F=23.74$, increasing steeply thereafter. Hence, performance did not decrease with inbreeding in the more highly inbred cows. Again, the conjecture is that highly inbred cows may be survivors of a purging process in which deleterious genes are being removed by selection.

Overall, the LOESS analyses indicated that additive models did not fit the data well. A parametric model including a cubic regression on $F$ provided the best fit 


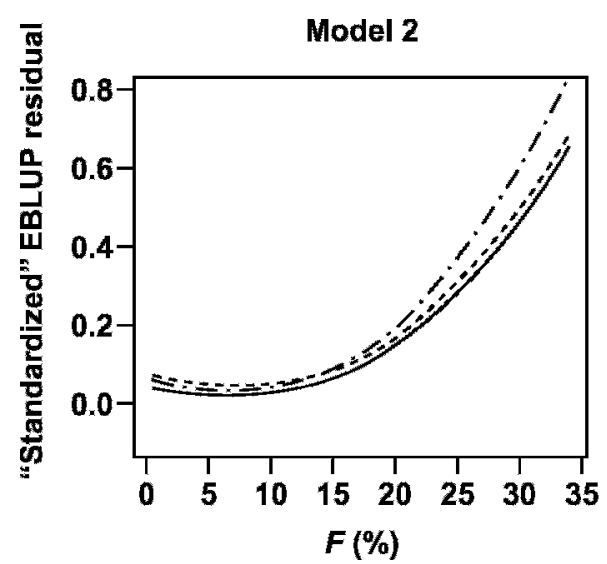

Model 3



Model 4



Figure 4. Robust LOESS curves for lactation milk (_- ), fat $(-\cdot-\cdot-)$, and protein (- - - ) yields based on empirical best linear unbiased predictions (EBLUP) residuals from models 2 to 4 (including $F,+F^{2},+F^{3}$; second-degree local polynomial and spanning parameter $f=1$ ). $F=$ inbreeding coefficient.

for all traits. Because this is an observational study (as opposed to a randomized trial), and the data were sparse at high levels of $F$, results from this study do not allow stronger inference.

Furthermore, before editing, most animals had pedigrees traceable until the $1950 \mathrm{~s}$, but only the subset used in the analyses had at least 6 generations of known pedigree. Perhaps the animals from this study accumulated their genomes, on average, through more generations of inbred ancestors from the base than an average cow in the population, thereby giving more opportunity for selection to operate against alleles with deleterious effects. Thus, results may not reflect a general population pattern.

\section{CONCLUSIONS}

In the sample of Jersey cows studied, inbreeding depression on yield traits was nonlinear. Yields tended not to decrease for $F \leq 7 \%$, and there was no additional depression in fat yield for $F>20 \%$, whereas protein yield seemed to stabilize at the higher levels of inbreeding. The joint effects of selection and of inbreeding depression could not be disentangled in this observational study. Cows with $\geq 6$ generations of complete pedigree data may have accumulated inbreeding through a greater number of generations, possibly giving selection more chances to purge deleterious alleles. Somatic cell scores did not exhibit detectable inbreeding depression, suggesting the absence of detectable dominance.

Including $F$ as a covariate in the linear model appeared to improve fit at small values of $F$, but it did not alleviate the lack of fit at higher levels of inbreeding. Standardized yield residuals increased with $F>10 \%$ for models with first- and second-order regressions on $F$, whereas third-order regressions on inbreeding provided an adequate fit to the data. These results should be interpreted with caution because the data were scarce at high levels of $F$.

\section{ACKNOWLEDGMENTS}

This research was supported by the American Jersey Cattle Association, by the Wisconsin Agriculture Experiment Station, and by grant NSF DEB-0089742. The USDA Animal Improvement Programs Laboratory and the American Jersey Cattle Association are acknowledged for providing the data.

\section{REFERENCES}

Allaire, F. R., and C. R. Henderson. 1965. Inbreeding within an artificially bred dairy cattle population. J. Dairy Sci. 48:1366-1371.

Bulmer, M. G. 1980. The Mathematical Theory of Quantitative Genetics. Oxford University Press, Oxford, UK.

Charlesworth, D., and B. Charlesworth. 1987. Inbreeding depression and its evolutionary consequences. Ann. Rev. Ecol. Syst. 18:237-268.

Cleveland, W. S. 1979. Robust locally weighted regression and smoothing scatterplots. J. Am. Stat. Assoc. 74:829-836.

Cleveland, W. S., and S. J. Devlin. 1988. Locally weighted regression: An approach to regression analysis by local fitting. J. Am. Stat. Assoc. 83:596-610.

Cleveland, W. S., E. Grosse, and W. M. Shyu. 1992. Local regression models. Pages 309-376 in Statistical Models in S. J. M. Chambers and T. J. Hastie, ed. S. Wadsworth and Brooks/Cole, Pacific Grove, CA.

Cleveland, W. S., and C. L. Loader. 1996. Smoothing by local regression: Principles and methods. Pages 10-49 in Statistical Theory and Computational Aspects of Smoothing. W. Haerdle and M. G. Schimek, ed. Springer, New York, NY.

Crow, J. F., and M. Kimura. 1970. An Introduction to Population Genetics Theory. Alpha Editions, Burgess International Group Inc., New York, NY.

Efron, B. 2005. Bayesians, frequentists, and scientists. J. Am. Stat. Assoc. 100:1-5. 
Falconer, D. S., and T. F. C. Mackay. 1996. Introduction to Quantitative Genetics. 4th ed. Longman, Essex, UK.

Fox, J. 2005. Introduction to Nonparametric Regression [Lecture notes]. http://socserv.socsci.mcmaster.ca/jfox/Courses/Oxford/ Accessed Feb. 14, 2005.

Glemin, S. 2003. How are deleterious mutations purged? Drift versus nonrandom mating. Evolution: Int. J. Org. Evolution 57:26782687.

Gulisija, D., and J. F. Crow. Inferring purging from pedigree data. Evolution: Int. J. Org. Evolution (accepted)

Gulisija, D., D. Gianola, K. A. Weigel, and M. A. Toro. 2006. Betweenfounder heterogeneity in inbreeding depression for production in Jersey cows. Livest. Sci. 104:244-253.

Hedrick, P. W. 1994. Purging inbreeding depression and the probability of extinction: Full-sib mating. Heredity 73:363-372.

Hill, W. G. 1982. Dominance and epistasis as components of heterosis. Z. Tierz. Zuchtungsbio. 99:161-168.

Hudson, G. F., and L. D. Van Vleck. 1984. Inbreeding of artificially bred dairy cattle in the northeastern United States. J. Dairy Sci. $67: 161-170$

Klemetsdal, G. 1998. The effect of inbreeding on racing performance in Norwegian cold-blooded trotters. Genet. Sel. Evol. 30:351-366.

Lynch, M. 1991. The genetic interpretation of inbreeding depression and outbreeding depression. Evolution: Int. J. Org. Evolution 45:622-629.

Lynch, M., and B. Walsh. 1998. Genetics and Analysis of Quantiative Traits. Sinauer Associates, Inc., Sunderland, MA.

Mackay, T. F. C. 2001. The genetic architecture of quantitative traits. Annu. Rev. Genet. 35:303-339.

Malécot, G. 1948. Les mathématiques de l'hérédité. Masson, Paris, France.

Miglior, F., E. B. Burnside, and W. D. Hohenboken. 1994. Heterogeneity among families of Holstein cattle in inbreeding depression for production traits. Pages 479-482 in Proc. 5th WCGALP XVIII. Guelph, ON, Canada.
Miglior, F., E. B. Burnside, and J. C. M. Dekkers. 1995a. Nonadditive genetic effects and inbreeding depression for somatic cell counts of Holstein cattle. J. Dairy Sci. 78:1168-1173.

Miglior, F., E. B. Burnside, and B. W. Kennedy. 1995b. Production traits of Holstein cattle: estimation of nonadditive genetic variance components and inbreeding depression. J. Dairy Sci. 78:1174-1180.

Miglior, F., B. Szkotnicki, and E. B. Burnside. 1992. Analysis of levels of inbreeding and inbreeding depression in Jersey cattle. J. Dairy Sci. 75:1112-1118.

R Development Core Team. 2004. R: A Language and Environment for Statistical Computing. R Foundation for Statistical Computing, Vienna, Austria. http://www.R-project.org Accessed June 1, 2004.

SAS Institute. 2002. SAS Procedure Guide, Version 9. SAS Inst. Inc., Cary, NC. http://support.sas.com/documentation/onlinedoc/ sas9doc.html Accessed Aug. 10, 2002.

Smith, L. A., B. G. Cassell, and R. E. Pearson. 1998. The effects of inbreeding on the lifetime performance of dairy cattle. J. Dairy Sci. 81:2729-2737.

Thompson, J. R., R. W. Everett, and N. L. Hammerschmidt. 2000a. Effects of inbreeding on production and survival in Holsteins. J. Dairy Sci. 83:1856-1864.

Thompson, J. R., R. W. Everett, and C. W. Wolfe. 2000b. Effects of inbreeding on production and survival in Jerseys. J. Dairy Sci. 83:2131-2138.

VanRaden, P. M., T. J. Lawlor, T. H. Short, and I. Hoeschele. 1992. Use of reproductive technology to estimate variances and predict effects of gene interactions. J. Dairy Sci. 75:2892-2901.

Wall, E., S. Brotherstone, J. F. Kearney, J. A. Woolliams, and M. P. Coffey. 2005. Impact of nonadditive genetic effects in the estimation of breeding values for fertility and correlated traits. J. Dairy Sci. 88:376-385.

Wright, S. 1922. Coefficients of inbreeding and relationship. Am. Nat. 56:330-338 